\title{
RESPOSTA DE PORTA-ENXERTOS DE CITROS AO DÉFICIT HÍDRICO ${ }^{1}$
}

\author{
ELAINE COSTA CERQUEIRA², MANOEL TEIXEIRA DE CASTRO NETO³, CLÓVIS PEREIRA PEIXOTO ${ }^{4}$, WALTER \\ DOS SANTOS SOARES FILHO ${ }^{3}$, CARLOS ALBERTO DA SILVA LEDO ${ }^{3}$, JUTAIR GARCIA DE OLIVEIRA ${ }^{5}$
}

\begin{abstract}
RESUMO - O trabalho foi conduzido em casa de vegetação na Embrapa Mandioca e Fruticultura, em Cruz das Almas - BA, e objetivou-se à identificação de porta-enxertos de citros melhor adaptados ao ecossistema de Tabuleiros Costeiros, quanto a tolerância à seca. Foram estudados os limoeiros 'Cravo' e 'Volkameriano', laranjeira 'Azeda' e os híbridos trifoliados HTR - 051, TSK x CTTR - 002 e TSK x CTTR - 017. O delineamento experimental foi o inteiramente casualizado, em esquema fatorial 6 õ 9, com seis genótipos e nove tempos de avaliação. As avaliações foram realizadas na sequência: irrigação, déficit hídrico e irrigação, quando plantas possuíam de 3 a 4 pares de folhas. Foram analisadas as variáveis massas secas da raiz e da parte aérea, o potencial hídrico da planta e a transpiração da folha. Quanto à massa seca da parte aérea, os genótipos limoeiro Volkameriano e laranjeira 'Azeda' apresentaram decréscimos no período de déficit hídrico, sendo que os demais não apresentaram diferenças significativas. Na ausência de irrigação, todos os genótipos apresentaram decréscimos nos seus potenciais hídricos, enquanto apenas os híbridos apresentaram recuperação, mantendo a transpiração. Os híbridos HTR - 051 e TSK x CTTR - 017 apresentaram os melhores desempenhos para todas as variáveis estudadas, mostrando-se mais promissores como porta-enxertos de citros em condições de déficit hídrico.
\end{abstract}

Termos para indexação: estresse hídrico, transpiração, tolerância à seca.

\section{RESPONSE OF CITRUS ROOTSTOCKS TO WATER DEFICIT}

\begin{abstract}
This work was carried out under green house condition at the Embrapa Cassava and Tropical Fruit Crops, Cruz das Almas - BahiaBrazil, aiming to identify citrus rootstocks better adapted to Coastal Table Land ecosystem as for their drought tolerance. It was studied the Rangpur lime, Volkamer lemon, Sour orange and the trifoliated hybrids HTR - 051, TSK x CTTR - 002, and TSK x CTTR - 017, the last three from the breeding program of the Embrapa Cassava and Tropical Fruits Crops. The experimental design was completely randomized under a 6 x 9 factorial, with six genotypes and nine period of evaluations. The evaluations followed the sequence: irrigation, water deficit, and irrigation when the plants had two to three leaves pairs. It was analyzed the variables root dry matter, dry matter of above ground parts, plant water potential, and leaf transpiration. For the above ground dry matter the genotypes Volkamer lemon and Sour orange showed decrease during the water deficit condition. The other genotypes showed no significant differences for this variable. Without irrigation, all genotypes decreased in their water potentials. The hybrids kept it unchanged maintaining increased transpiration rates. The hybrids HTR - 051, and TSK x CTTR - 017 presented the best performances for all variables studied being more promising as rootstocks for water deficit conditions.
\end{abstract}

Index terms: water stress, transpiration, drought tolerance.

\section{INTRODUÇÃO}

Embora o Brasil se destaque como maior produtor mundial de citros e maior produtor e exportador de suco concentrado congelado de laranja, deve-se considerar a grande diversificação agroclimática do País e suas condições particulares de cultivo, compreendendo uma multiplicidade de ambientes, nos quais se verifica desde a ocorrência de geadas ocasionais ou freqüentes e até estresses hídricos ou térmicos, implicando múltiplas floradas com crescimento e maturação irregulares dos frutos.

A citricultura baiana está concentrada no ecossistema de Tabuleiros Costeiros, onde são registradas precipitações pluviais anuais de $1.000 \mathrm{~mm}$ a $1.200 \mathrm{~mm}$, distribuídas em 8 a 10 meses, com dois a seis meses de deficiência hídrica, sendo o uso de irrigação praticamente nulo. O estresse é considerado como um desvio significativo das condições ótimas para a vida, induzindo mudanças e respostas em todos os níveis funcionais do organismo, as quais, em princípio, podem ser reversíveis, mas podem tornar-se permanentes (Larcher, 2000).

Entre os vários fatores limitantes da produção vegetal, o déficit hídrico ocupa posição de destaque, pois além de afetar as relações hídricas nas plantas, alterando-lhes o metabolismo, é fenômeno que ocorre em grandes extensões de áreas cultiváveis (Nogueira et al., 2001). Sendo o cultivo dos citros no Brasil predominantemente sem irrigação, é desejável que se usem combinações copa/porta-enxerto que mostrem, entre outras características de interesse agronômico, tolerância à seca, em vista da ocorrência de déficits hídricos temporários em várias regiões citrícolas (Ortolani et al., 1991). Para combinações com as mesmas copas, as diferentes variedades de porta-enxertos afetam, distintamente, as relações hídricas e as trocas gasosas (Castle et al., 1989).

A vulnerabilidade da citricultura baiana e nordestina em geral é muito grande, pela presença quase única da combinação laranjeira 'Pêra' [Citrus sinensis (L.) Osb.] / limoeiro 'Cravo' (C. limonia Osb.) na sustentação dos pomares, tornando urgente um programa de diversificação de variedades. O emprego de um único porta-enxerto para todas as variedades - copa não permite, provavelmente, atender ao máximo potencial inerente a cada variedade, impedindo que a planta manifeste toda sua capacidade produtiva, além de se constituir em inconveniente problema no caso de moléstias endêmicas (Pompeu Junior, 1991).

De acordo com Nogueira et al. (2001), genótipos que apresentem diversidade na resposta ao estresse hídrico constituem excelentes materiais para serem utilizados em programas de melhoramento genético. Vários caracteres fisiológicos podem ser empregados na avaliação das respostas das espécies vegetais ao estresse hídrico, destacando-se entre eles o potencial hídrico foliar, a condutância estomática e a transpiração.

Assim, este trabalho teve como objetivo a identificação de portaenxertos de citros (variedades e híbridos) que apresentem melhores respostas ao déficit hídrico, sendo relativamente tolerantes à seca, mostrando-se mais adaptados ao ecossistema de Tabuleiros Costeiros.

\section{MATERIAL E MÉTODOS}

Este estudo foi conduzido em casa de vegetação na Embrapa Mandioca e Fruticultura, localizada no município de Cruz das Almas BA. O material vegetal foi coletado no Banco Ativo de Germoplasma de Citros, compreendendo sementes dos híbridos tangerineira 'Sunki' ( $C$. sunki Hort. ex Tan.) õ citrange [C. sinensis õ Pocirus trifoliata (L.) Raf.]

\footnotetext{
${ }^{1}$ (Trabalho 059/2004). Recebido: 20/04/2004. Aceito para publicação: 06/10/2004.

${ }^{2}$ Engenheira Agrônoma. Mestranda em Ciências Agrárias/UFBA - ellainnep@ bol.com.br.

${ }^{3}$ Eng ${ }^{\circ}$ Agrônomo. PhD. Embrapa Mandioca e Fruticultura. Cruz das Almas-BA. castro@ cnpmf.embrapa.br.

${ }^{4}$ Eng $^{\text {o }}$ Agrônomo. DSc. Orientador. Escola de Agronomia da UFBA - cpeixot@ ufba.br.

${ }^{5}$ Estudante Acadêmico da AGRUFBA. Bolsista Pibic/CNPq.
} 
TABELA 1 - Médias $^{1}$ da massa seca da raiz (g) de porta-enxertos de citros avaliados sob irrigação, estresse hídrico e re-irrigação. Cruz das Almas - BA, 2004.

\begin{tabular}{|c|c|c|c|c|c|c|c|c|c|c|}
\hline \multirow{3}{*}{ Porta-enxerto $^{2}$} & \multicolumn{9}{|c|}{ Avaliações } & \multirow{3}{*}{ Médias } \\
\hline & \multicolumn{2}{|c|}{ Irrigado (dias) } & \multicolumn{3}{|c|}{ Déficit hídrico (dias) } & \multicolumn{4}{|c|}{ Irrigado (dias) } & \\
\hline & $\mathbf{0}$ & 4 & 4 & 8 & 12 & 1 & 2 & 3 & 4 & \\
\hline$\overline{\mathrm{LVK}}$ & $0,9 \mathrm{aC}$ & $1,1 \mathrm{aABC}$ & $1,2 \mathrm{aA}$ & $1,2 \mathrm{aA}$ & $1,1 \mathrm{aABC}$ & $1,1 \mathrm{aAB}$ & $1,1 \mathrm{aABC}$ & $1,1 \mathrm{aABC}$ & $0,9 \mathrm{bBC}$ & $1,1 \mathrm{a}$ \\
\hline LAZ & $0,8 \mathrm{aC}$ & $0,8 \mathrm{bBC}$ & 1,0abAB & $1,1 \mathrm{aA}$ & $1,0 \mathrm{aAB}$ & $1,0 \mathrm{aAB}$ & $1,2 \mathrm{aA}$ & $1,2 \mathrm{aA}$ & $1,1 \mathrm{aA}$ & $1,0 \mathrm{a}$ \\
\hline TSK x CTTR-002 & $0,5 \mathrm{bA}$ & $0,6 \mathrm{cA}$ & $0,6 \mathrm{cA}$ & $0,6 \mathrm{bcA}$ & $0,6 \mathrm{bcA}$ & $0,6 \mathrm{bcA}$ & $0,7 \mathrm{bcA}$ & $0,6 \mathrm{cA}$ & $0,5 \mathrm{cA}$ & $0,6 \mathrm{c}$ \\
\hline LCR & $0,8 \mathrm{aA}$ & $0,9 \mathrm{bA}$ & $0,9 \mathrm{bA}$ & $0,8 \mathrm{bA}$ & $0,7 \mathrm{bA}$ & $0,8 \mathrm{bA}$ & $0,8 \mathrm{bA}$ & $0,8 \mathrm{bA}$ & $0,8 \mathrm{bA}$ & $0,8 \mathrm{~b}$ \\
\hline HTR-051 & $0,3 \mathrm{bA}$ & $0,4 \mathrm{cA}$ & $0,4 \mathrm{cA}$ & $0,4 \mathrm{dA}$ & $0,4 \mathrm{cA}$ & $0,4 \mathrm{cA}$ & $0,4 \mathrm{dA}$ & $0,5 \mathrm{cA}$ & $0,4 \mathrm{cA}$ & $0,4 \mathrm{e}$ \\
\hline TSK x CTTR-017 & $0,3 \mathrm{bB}$ & $0,4 \mathrm{cAB}$ & $0,6 \mathrm{cA}$ & $0,5 \mathrm{cdAB}$ & $0,4 \mathrm{cAB}$ & $0,6 \mathrm{cdA}$ & $0,6 \mathrm{cdA}$ & $0,6 \mathrm{cA}$ & $0,5 \mathrm{cAB}$ & $0,5 \mathrm{~d}$ \\
\hline$\overline{\text { Médias }}$ & $0,6 \mathrm{~B}$ & $0,7 \mathrm{~A}$ & $0,8 \mathrm{~A}$ & $0,8 \mathrm{~A}$ & $0,7 \mathrm{~A}$ & $0,8 \mathrm{~A}$ & $0,8 \mathrm{~A}$ & $0,8 \mathrm{~A}$ & $0,7 \mathrm{~A}$ & \\
\hline
\end{tabular}

${ }^{1}$ Médias seguidas pela mesma letra minúscula nas colunas e maiúscula nas linhas não diferem estatisticamente entre si, pelo teste de Tukey, a 5\% de probabilidade.

$\mathrm{CV}(\%)=14,53$

'Troyer' (TSK x CTTR - 002), TSK õ CTTR - 017 e híbrido trifoliado HTR 051, obtidos pelo Programa de Melhoramento Genético de Citros da Embrapa Mandioca e Fruticultura, bem como das variedades limoeiro Volkameriano (C. volkameriana Ten. et Pasq.), limoeiro Cravo (LCR) (C. limonea Osb.) e laranjeira Azeda (LAZ) (C. aurantium L.). As sementes foram germinadas em uma mistura de substrato composto de Plantmax (Eucatex Agro®) e fibra de coco, na proporção de 1:1.

Plântulas dos diferentes genótipos estudados foram obtidas a partir de 300 sementes de cada indivíduo. Após a presença de dois pares de folhas permanentes, as plântulas foram transplantadas para vasos com capacidade de $300 \mathrm{~mL}$, contendo a mesma mistura usada para a germinação das sementes, utilizando-se de adubação complementar com $150 \mathrm{~g}$ de Osmocoat (19-6-10, Scolts ${ }^{\circledR}$ ) e de PgMix (14-16-18, Hydro $\left.{ }^{\circledR}\right)$ para cada $50 \mathrm{~kg}$ da mistura, sendo irrigadas a cada dois dias até o início do experimento, quando apresentavam de três a quatro pares de folhas definitivas, ocasião em que foram iniciadas as avaliações da massa seca (MS) da planta, do potencial hídrico (PH) e da transpiração foliar (T).

A massa seca foi obtida após secagem das frações vegetais (raiz e parte aérea) até peso constante, em estufa a $70+5^{\circ} \mathrm{C}$ com ventilação de ar forçado, sendo aferidas em balança analítica e os resultados expressos em gramas (g), por tempo de exposição tratamento. O potencial hídrico das plantas (-MPa) foi determinado pelo método da bomba de pressão (Salisbury e Ross, 1992). A transpiração foliar $\left(\mathrm{mol} \mathrm{cm} \mathrm{cm}^{-2} \mathrm{~s}^{-1}\right)$ foi determinada pelo método de pesagem da perda diária de água, isolando o recipiente, utilizando-se de saco plástico, vedando-o ao caule, de forma que a perda de água ocorreu apenas por transpiração, avaliando-se a cada duas horas, das 6 às 18 horas (Cerqueira, 2004).

O delineamento experimental foi o inteiramente casualizado, no esquema fatorial $6 \times 9$, com seis tratamentos (variedades e híbridos), sob diferentes regimes hídricos: com irrigação ( 0 e 4 dias) e sem irrigação, considerando-se diversos períodos de exposição ao estresse $(4 ; 8$ e 12 dias), seguido de tempo de recuperação ( $1 ; 2 ; 3$ e 4 dias), perfazendo um total de nove tempos de avaliação, com cinco repetições por tratamento. As irrigações foram feitas até a completa saturação do substrato.

\section{RESULTADOS E DISCUSSÃO}

Observa-se um comportamento diferenciado entre os genótipos com relação às variáveis estudadas, indicando uma variação nas respostas aos diferentes regimes hídricos.

Os acúmulos de massa seca nas frações vegetais raiz e parte aérea são apresentados nas Tabelas 1 e 2, respectivamente. Considerando a massa seca das raízes, observa-se que não houve diferenças significativas entre os regimes hídricos para todos os genótipos nas avaliações consecutivas. Após o déficit hídrico, a partir do segundo dia de irrigação, a LAZ apresentou incremento de $50 \%$, o que é uma característica desejável, pois um dos mecanismos de tolerância seca é justamente o desenvolvimento das raízes em busca de água. Observa-se que, na média (Tabela 1), o LVK e LAZ se mostraram superiores aos demais porta-enxertos, mesmo não diferindo entre si.

Quanto à produção de massa seca da parte aérea (Tabela 2), o LVK, aos 8 dias de déficit, apresentou uma queda de $20 \%$, em relação ao período irrigado, mantendo-se constante aos 12 dias e, em seguida, decrescendo mesmo após o período de recuperação. A LAZ só apresentou diferença significativa aos 12 dias de déficit, com decréscimo de $12 \%$, não havendo recuperação e continuando a decrescer. O LCR decresceu sob déficit hídrico, no entanto, apresentou um aumento de $27 \%$ após esse período, verificando-se diferença significativa entre esses tratamentos quando comparados aos 12 dias de déficit hídrico. Essa redução também foi observada em plantas de aroeira-do-sertão (Myracrodruon urundeuva Fr. All.) quando submetidas ao déficit hídrico, principalmente com o prolongamento do tempo de deficiência hídrica (Paim, 2002).

De maneira geral, os híbridos não apresentaram redução da massa seca da parte aérea com o déficit hídrico, nem lograram recuperação com o novo período irrigado. Dessa forma, não apresentaram sensibilidade ao déficit hídrico, mantendo a assimilação orgânica, mesmo em menores taxas, provavelmente devido aos seus menores portes e também por serem trifoliados, apresentando folíolos reduzidos.

TABELA 2 - Médias ${ }^{1}$ da massa seca da parte aérea (g) de porta-enxertos de citros avaliados sob irrigação, estresse hídrico e re-irrigação. Cruz das Almas - BA, 2004

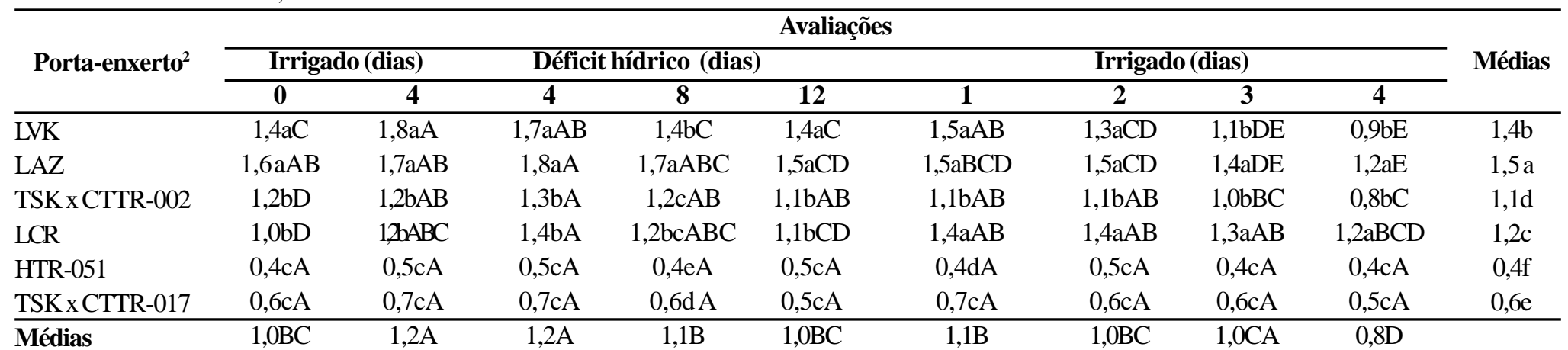

${ }^{1}$ Médias seguidas pela mesma letra minúscula nas colunas e maiúscula nas linhas não diferem estatisticamente entre si, pelo teste de Tukey, a 5\% de probabilidade. $\mathrm{CV}(\%)=10,80$ 
TABELA 3 - Médias ${ }^{1}$ do potencial hídrico (-MPa) de porta-enxertos de citros avaliados sob irrigação, estresse hídrico e re-irrigação. Cruz das Almas - BA, 2004.

\begin{tabular}{|c|c|c|c|c|c|c|c|c|c|c|}
\hline \multirow[b]{2}{*}{ Porta-enxerto $^{2}$} & \multicolumn{9}{|c|}{ Avaliações } & \multirow[b]{2}{*}{ Médias } \\
\hline & \multicolumn{2}{|c|}{ Irrigado (dias) } & \multicolumn{3}{|c|}{ Déficit hídrico (dias) } & \multicolumn{4}{|c|}{ Irrigado (dias) } & \\
\hline$\overline{\mathrm{LVK}}$ & $0,8 \mathrm{aC}$ & $0,6 \mathrm{aC}$ & $0,9 \mathrm{aC}$ & $2,7 \mathrm{aB}$ & $3,3 \mathrm{aA}$ & $3,0 \mathrm{aAB}$ & $2,7 \mathrm{bB}$ & $2,7 \mathrm{aAB}$ & $2,6 \mathrm{aB}$ & $2,1 \mathrm{a}$ \\
\hline TSK x CTTR-002 & $0,8 \mathrm{aBC}$ & $0,7 \mathrm{aC}$ & $0,9 \mathrm{aBC}$ & $1,3 \mathrm{cB}$ & $3,1 \mathrm{aA}$ & $1,3 \mathrm{bBC}$ & $1,3 \mathrm{cB}$ & $1,3 \mathrm{bB}$ & $1,2 \mathrm{bBC}$ & $1,3 b c$ \\
\hline LCR & $0,7 \mathrm{aE}$ & $0,6 \mathrm{aE}$ & $1,0 \mathrm{aDE}$ & $2,7 \mathrm{aBC}$ & $3,2 \mathrm{aA}$ & $3,2 \mathrm{aAB}$ & $3,3 \mathrm{aA}$ & $2,5 \mathrm{aC}$ & $1,5 \mathrm{bD}$ & $2,1 \mathrm{a}$ \\
\hline HTR-051 & $0,8 \mathrm{aB}$ & $0,8 \mathrm{aB}$ & $0,9 \mathrm{aB}$ & $1,1 \mathrm{cAB}$ & $1,5 \mathrm{cA}$ & $1,2 \mathrm{bAB}$ & $1,2 \mathrm{cAB}$ & $1,3 \mathrm{bAB}$ & $1,2 \mathrm{bAB}$ & $1,1 \mathrm{~d}$ \\
\hline
\end{tabular}

${ }^{1}$ Médias seguidas pela mesma letra minúscula nas colunas e maiúscula nas linhas não diferem estatisticamente entre si, pelo teste de Tukey, a 5\% de probabilidade. $\mathrm{CV}(\%)=17,80$

Na Tabela 3, observa-se que a variável potencial hídrico $\left(\emptyset_{\mathrm{w}}\right)$ não apresentou diferenças significativas entre os porta-enxertos, quando irrigados, e aos 4 dias de déficit hídrico. Aos 8 dias de suspensão da irrigação, verificaram-se diferenças significativas entre os genótipos avaliados, sendo que os limoeiros 'Cravo' e 'Volkameriano' não diferiram entre si e foram os que aprestaram valores de $\emptyset_{w}$ mais negativos no período de déficit hídrico, com decréscimo de $350 \%$ em relação ao período irrigado. Por outro lado, os híbridos obtidos pelo Programa de Melhoramento Genético de Citros da Embrapa Mandioca e Fruticultura manifestaram menor sensibilidade nesse período, com os valores de potenciais de água menos reduzidos, enquanto a laranjeira 'Azeda', com uma redução de $190 \%$, mostrou comportamento intermediário. Aos 12 dias de déficit hídrico, todos os genótipos tiveram redução no potencial hídrico, sendo que os híbridos HTR - 051 (87\%) e TSK x CTTR - 017 (175\%) foram os menos sensíveis. O limoeiro 'Volkameriano' (450\%) foi o que apresentou potencial mais negativo, indicativo de sua maior sensibilidade.

Medina et al. (1999) verificaram, em plantas de citros submetidas à deficiência hídrica, que os potenciais da água nas folhas decresceram progressivamente em função do número de dias sem irrigação, constatando após nove dias de déficit potenciais ao redor de -2,0 e -2,5 MPa em laranjeiras-doces ( $C$. sinensis) enxertadas em limoeiro 'Cravo' e em $P$. trifoliata, respectivamente. Observou-se em todos os genótipos (variedades e híbridos) submetidos à deficiência hídrica uma redução no potencial de água, com decréscimos progressivos em função do número de dias sem irrigação, fato este também observado por Brunini \& Cardoso (1998), em plantas de seringueira (Hevea brasiliensis Muell.Arg.). Os porta-enxertos limoeiros 'Cravo' e 'Volkameriano' apresentaram valores de potencial hídrico de -3,2 e -3,3 MPa, respectivamente, semelhantes aos encontrados por Calbo \& Moraes (1997) em plantas de buriti (Mauritia vinifera Mart.), estando os mesmos, portanto, na faixa de valores de potenciais hídricos esperados em plantas mesófitas cultivadas.

A laranjeira 'Azeda' e o híbrido TSK x CTTR - 002 demonstraram uma recuperação logo no primeiro dia da irrigação (acréscimo de $60 \%$ e $58 \%$, respectivamente), não apresentando diferenças estatísticas entre as avaliações seguintes. Quanto ao limoeiro 'Cravo', sua recuperação foi lenta após a irrigação, constatando-se efeitos da reidratação só a partir do terceiro dia com um acréscimo de $22 \%$ e, no quarto dia, com um acréscimo de $53 \%$.

O híbrido TSK x CTTR - 017 apresenta acréscimo no potencial hídrico logo no primeiro dia do período da recuperação (47\%) sem diferenças significativas nas demais avaliações. Destaca-se o comportamento do híbrido HTR - 051 que, apesar de apresentar apenas $21 \%$ de recuperação, foi o genótipo menos sensível durante a fase de déficit hídrico, enquanto TSK x CTTR - 002, embora seu potencial tenha sido bastante reduzido aos 12 dias sem irrigação, exibiu grande poder de recuperação (58\%), demonstrando potencial para suportar graus mais avançados de desidratação, em situações de ocorrência de déficits hídricos.

A perda de água pelas plantas ocorre principalmente em função da transpiração estomática, pois os estômatos constituem a via de escape que menor resistência oferece à difusão gasosa. A intensidade dessa transpiração varia com a ação de fatores internos (área superficial, distribuição e número dos estômatos) e de fatores externos (luz, umidade do ar, temperatura e disponibilidade de água no solo). Pode ser controlada pela planta, que consegue aumentar ou diminuir a perda de água, dependendo do ambiente em que se encontra. Como a abertura dos estômatos depende do grau de saturação hídrica das células estomáticas, pode haver grande restrição da transpiração quando o déficit de água na planta for muito grande.

Neste trabalho (Figura 1), a transpiração foi claramente afetada pelos regimes hídricos aos quais as plantas foram submetidas, registrando-se uma queda acentuada dessa variável a partir da primeira avaliação sem irrigação, uma vez que, sob condições de deficiência hídrica, o fluxo interno de água na folha se torna insuficiente para a manutenção da turgescência foliar, atuando diretamente no declínio da condutância estomática e da transpiração (Larcher, 2000).

Considerando os tratamentos hídricos aplicados, o limoeiro 'Volkameriano' e a laranjeira 'Azeda' (Figura 1) mostraram variações semelhantes, observando-se queda progressiva nos valores da transpiração diária entre o período irrigado e não-irrigado. Cabe acrescentar, contudo, que o limoeiro 'Volkameriano' não apresentou recuperação da transpiração após a irrigação, diferentemente da laranjeira 'Azeda', que manifestou uma recuperação de $50 \%$ aos 4 dias após a irrigação.

O híbrido TSK x CTTR - 002 apresentou, no período de déficit hídrico, um decréscimo relativamente mais lento da transpiração, sendo mais intenso aos 12 dias de déficit. No período de recuperação, notou-se um aumento de sua transpiração, com maiores valores nas horas mais quentes do dia, fato este verificado para todos os genótipos estudados, embora com diferentes intensidades.

O limoeiro 'Cravo' mostrou um decréscimo da transpiração logo nos primeiros 4 dias de déficit hídrico, mantendo esse status hídrico por todo o período de déficit, manifestando uma pequena recuperação apenas no quarto dia após a irrigação, o que certamente prejudicou suas trocas gasosas.

O híbrido HTR - 051 manteve a taxa de transpiração equivalente à do período irrigado até o quarto dia de déficit hídrico, registrando queda somente a partir do oitavo dia e mantendo-a por todo o restante do período de estresse. Após a irrigação, a recuperação da transpiração iniciou-se no primeiro dia, sendo mantida em valores semelhantes aos verificados para as plantas com irrigação diária, sugerindo que houve uma efetiva recuperação de sua transpiração, com restauração plena das trocas gasosas e, possivelmente, manutenção do processo fotossintético. Resultados de recuperação após o déficit hídrico também foram relatados por Calbo e Moraes (1997) em plantas de buriti, no primeiro dia de reidratação.

Relativamente à variação da transpiração apresentada pelo híbrido TSK x CTTR - 017, este, apesar de sofrer os efeitos do déficit de água, não mostrou grande intensidade aos 4 e 8 dias de déficit, 

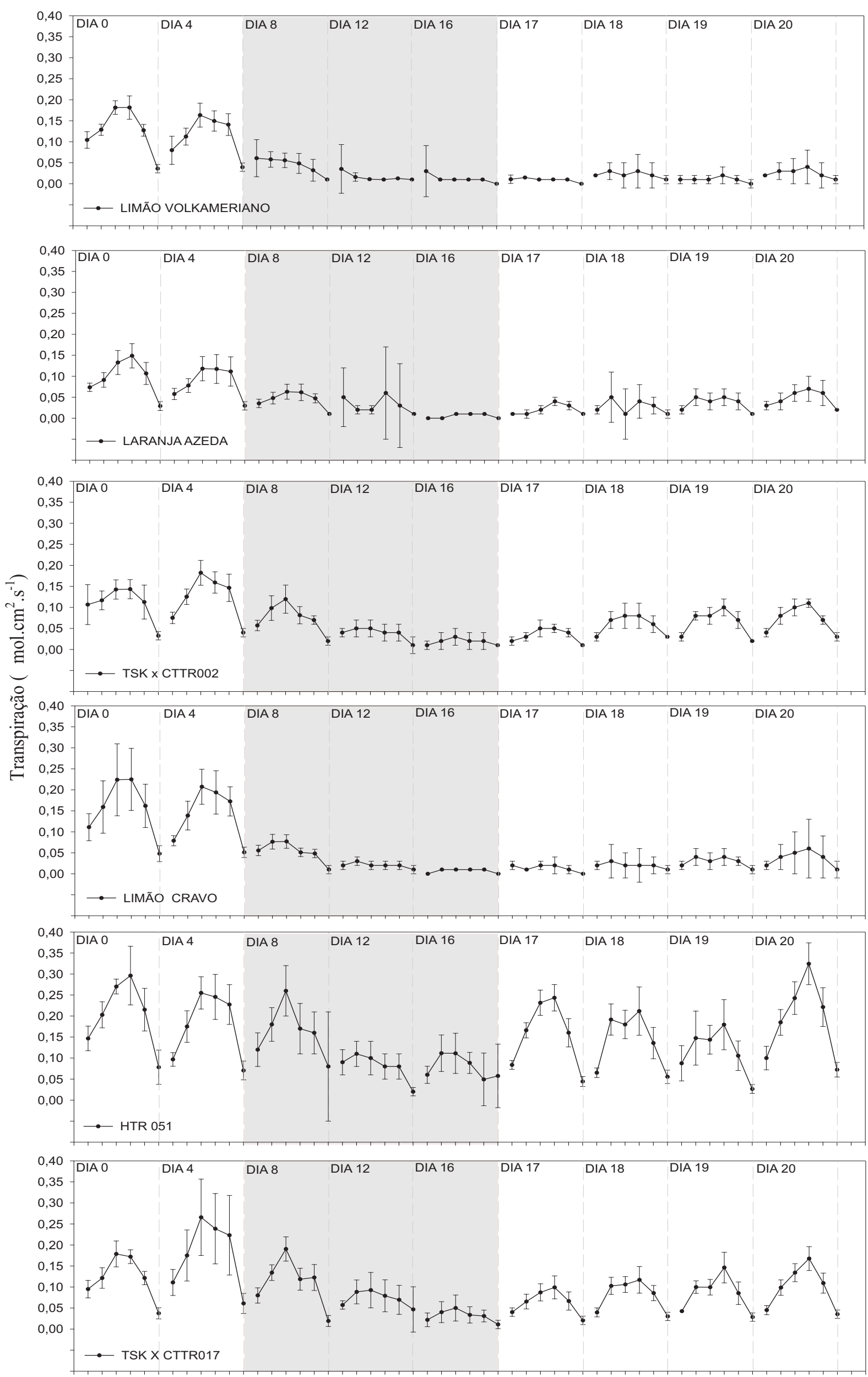

810121416188101214161881012141618810121416188101214161881012141618810121416188101214161881012141618810 HORA DO DIA (horas)

FIGURA 1 - Transpiração do limoeiro 'Volkameriano' (Citrus volkameriana Ten. et Pasq.), laranjeira 'Azeda' (C. aurantium L.), híbrido TSK [tangerina 'Sunki' (C. sunki Hort. ex Tan.)] x CTTR \{citrange 'Troyer' [C. paradisi Macf. x Poncirus trifoliata (L.) Raf.] -002 , limoeiro 'Cravo' (C. limonia Osb.), híbrido trifoliado HTR - 051, híbrido TSK x CTTR - 017 considerando diferentes regimes hídricos (irrigado: 0 e 4 dias; déficit hídrico com suspensão da hidratação: 4; 8 e 12 dias; e re-irrigação: 1; 2; 3 e 4 dias), Cruz das Almas - BA, 2004.

Barras representam o desvio-padrão. 
intensificando-se aos 12 dias. Após a reidratação, verificou-se sua recuperação, embora em menores taxas que a do HTR - 051, mas com bom indicativo de tolerância a períodos de estiagem, havendo evidências da recuperação de seu crescimento, indicada pela manutenção dos processos de trocas gasosas.

Por esses resultados, considerando-se o conjunto de genótipos avaliados, pode-se inferir que os híbridos TSK õ CTTR - 002, TSK õ CTTR - 017 e HTR - 051 apresentam maior tolerância ou mesmo maior capacidade de recuperação quando submetidos ao déficit hídrico seguido de hidratação, uma vez que mantiveram, dentro das condições deste estudo, seus processos de trocas gasosas através da transpiração. Resultados semelhantes aos verificados neste trabalho foram observados por Medina et al. (1999) e Nogueira et al. (2000) para laranjeira-doce e em outras espécies, como a acerola (Malpighia emarginata D.C.), respectivamente.

\section{CONCLUSÕES}

Considerando-se o conjunto de genótipos avaliados e as condições experimentais observadas neste estudo, pôde-se chegar às seguintes conclusões:

1) Os híbridos HTR - 051 e TSK õ CTTR - 017 apresentam maior tolerância à desidratação, o que indica seu potencial de uso como portaenxertos de citros mais promissores em condições de déficit hídrico.

2) Os híbridos HTR - 051, TSK õ CTTR - 017 e TSK õ CTTR 002, além da laranjeira 'Azeda', manifestam maior poder de recuperação após o déficit hídrico, sendo capazes de manter as trocas gasosas e a produção de matéria seca.

3) Os limoeiros 'Volkameriano' e 'Cravo' mostram-se mais sensíveis ao déficit hídrico, apresentando maior redução do potencial hídrico e baixa capacidade de recuperação.

\section{REFERÊNCIAS}

BRUNINI, O.; CARDOSO, M. Efeito do déficit hídrico no solo sobre o comportamento estomático e potencial da água em mudas de seringueira. Pesquisa Agropecuária Brasileira, Brasília, v.33, n.7, p.1053-1060, 1998.

CALBO, M. E. R.; MORAES, J. A. P. V. de. Fotossíntese, condutância estomática, transpiração e ajustamento osmótico de plantas de buriti submetidas a estresse hídrico. Revista Brasileira de Fisiologia Vegetal, Londrina, v. 9, n.2, p.117-123, 1997.

CASTLE, W.S.; TUCKER, D.P.H.; KREZDORN, A.H.; YOTSEY, C. O. Rootstocks for Florida Citrus. Gainesville: Institute of Food and Agricultural Science, University of Florida, 1989. 47p.

CERQUEIRA, E. C. Avaliação de porta-enxerto de citros visando tolerância ao déficit hídrico. 2004. 57f. Dissertação (Mestrado em Ciências Agrárias), Eescola de Agronomia, Universidade Federal da Bahia, Cruz das Almas, 2004.

LARCHER, W. Ecofisiologia vegetal. São Paulo: Rima, 2000. 531p.

MEDINA, C. L.; MACHADO, E. C.; GOMES, M. M. Condutância estomática, transpiração e fotossíntese em laranja 'Valência' sob deficiência hídrica. Revista Brasileira de Fisiologia Vegetal, Londrina, v.11, n.1, p.29-34, 1999.

NOGUEIRA, R. J. M. C.; MORAES, J. A. P. V.; BURITY, H. A. Alterações na resistência à difusão de vapor das folhas e relações hídricas em aceroleira submetidas a déficit de água. Revista Brasileira de Fisiologia Vegetal, Londrina, v.13, n.1, p.75-87, 2001.

ORTOLANI, A.; PEDRO JUNIOR, M.J.; ALFONSI, R.R. Agroclimatologia e o cultivo de citros. In: RODRIGUES, O.; VIÉGAS, F.; POMPEU JUNIOR, J.; AMARO, A. A. (Ed.) Citricultura brasileira. Campinas: Fundação Cargill, 1991. p.153-195.

PAIM, A. C. B. Avaliação do efeito do estresse hídrico na estrutura, ecofisiologia e na bioquímica de plântulas Myracrodruon urundeuva Fr. All. (ANACARDIACEAE), 2002. 80f. Dissertação (Mestrado em Botânica), Universidade Estadual da Bahia, Feira de Santana, 2002.

POMPEU JUNIOR, J. Porta-enxertos. In: RODRIGUES, O.; VIEGAS, F.; POMPEU JUNIOR, J.; AMARO, A. A. Citricultura brasileira. Campinas: Fundação Cargill, 1991. v.1, p.264-280.

SALISBURY, F. B.; ROSS, C. W. Plant Physiology. $4^{\text {th }}$ ed. California: Wadsworth, 1992.747p. 